\title{
Multiple Neurofibromas with Hypertrichosis in a Filipino Patient with Neurofibromatosis 1
}

\author{
Leah Antoinette M. Caro-Chang, ${ }^{1}$ Maria Franchesca S. Quinio ${ }^{1}$ and Georgina C. Pastorfide ${ }^{2}$ \\ ${ }^{1}$ Section of Dermatology, Department of Medicine, Philippine General Hospital, University of the Philippines Manila \\ ${ }^{2}$ Section of Dermatology, Department of Medicine, College of Medicine and Philippine General Hospital, University of the Philippines Manila
}

\begin{abstract}
This is a case of an 11-year-old male who presented with multiple neurofibromas with hypertrichosis. Classic cutaneous neurofibromas, café-au-lait macules, axillary freckling, Lisch nodules, and scoliosis were also present fulfilling a diagnosis of Neurofibromatosis type 1. This is the first report of multiple neurofibromas with hypertrichosis in the Philippines. Hypertrichosis overlying a neurofibroma is rarely reported and the mechanism remains to be elucidated.
\end{abstract}

Key Words: neurofibromatosis 1, hypertrichosis, neurofibromas

\section{INTRODUCTION}

Neurofibromatosis type 1 (NF 1 ) is a genodermatosis caused by mutations in the NF 1 gene that encodes the tumor suppressor protein neurofibromin. ${ }^{1}$ This autosomal dominant disorder affects approximately 1 in 4,000 individuals. $^{2}$ This disease can be diagnosed clinically with cutaneous neurofibromas as a hallmark finding. These usually present as skin-colored papules or nodules. This report presents a patient who presented with typical cutaneous neurofibromas but also presented with multiple patches with hypertrichosis that were histopathologically consistent with neurofibromas.

\section{CASE}

An 11-year-old male consulted for multiple brown patches on the trunk with overlying hair. At birth, the patient had a dark brown to black patch at the lumbosacral portion of his back. It remained stable in size and asymptomatic but developed thin hair after four years. At that time, the mother also noted the appearance of multiple skin-colored papules on his upper back and multiple brown patches with thin hair all over the trunk. These increased in number but remained asymptomatic. They consulted several

E-poster presented at the $41^{\text {st }}$ Annual Convention of the Philippine Dermatological Society, November 7-9, 2018, EDSA Shangri-La Hotel, Mandaluyong City, Philippines

Corresponding author: Leah Antoinette M. Caro-Chang, MD Section of Dermatology

Department of Medicine

Philippine General Hospital

University of the Philippines Manila

Taft Avenue, Manila 1000, Philippines

Telephone: +632 5548400 local 5105/5106

Email: Imcaro@up.edu.ph dermatologists and was managed as pityriasis versicolor and hypersensitivity reaction with unrecalled creams but all lesions persisted. Biopsy of the lesions was never done.

The patient was born full-term to a 26-year-old G1P1 mother without any feto-maternal complications. Immunization was complete and no other medical conditions were present. There was no history of seizures. The patient's development was at par with age but performed below average at school due to difficulty in reading. There were no family members with similar lesions. 
On examination, there were multiple skin-colored papules on the trunk extending to the thighs (Figures 1 and 2). There were multiple well-defined brown macules and patches located on the arms, abdomen, and back (Figures 3 and 4). There was axillary freckling (Figure 5). There was a black patch with hypertrichosis located midline at the lumbosacral region (Figure 6). Dermoscopy showed a brown reticular pattern with perifollicular hypopigmentation and terminal hair (Figure 7). There were multiple brown, slightly erythematous macules with overlying hypertrichosis on the trunk (Figure 8). Dermoscopy also revealed a reticulated brown pattern, perifollicular hypopigmentation but with some areas of erythema, and terminal hair growth (Figure 9).
Biopsy of three lesions was done. The first lesion was a skin-colored papule on the back which showed a wellcircumscribed, unencapsulated proliferation of spindleshaped cells with wavy nuclei arranged in an orderly fashion in a pale mucinous stroma surrounding adnexal structures, consistent with a neurofibroma (Figure 10). The black patch with overlying hair at the lumbosacral region (Figure 11) and the brown macules also with overlying hair on the abdomen (Figure 12) also revealed the same proliferation of spindle-shaped cells with wavy nuclei in a pale mucinous stroma with the former showing basal hyperpigmentation. Despite varying clinical presentations, all these lesions were histopathologically consistent with neurofibromas.

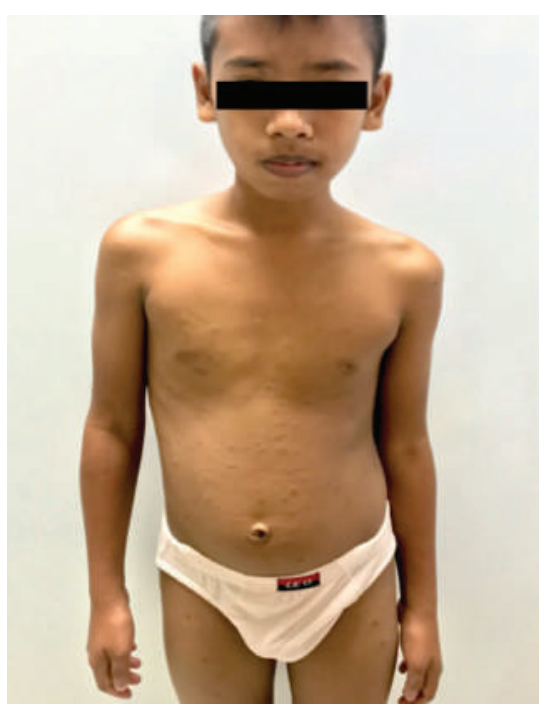

Figure 1. There were skin-colored papules on the trunk extending to the thighs.

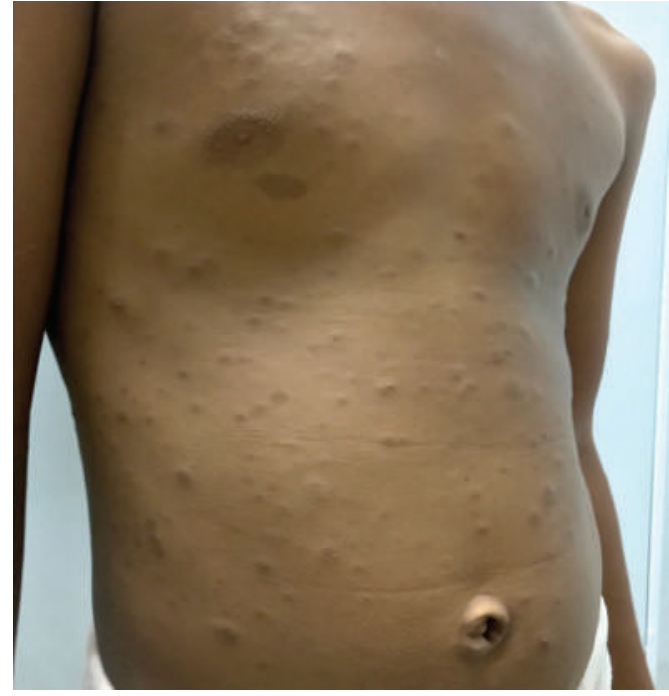

Figure 2. Closer view reveals multiple skincolored papules, brown plaques and brown patches (café-au-lait macules).
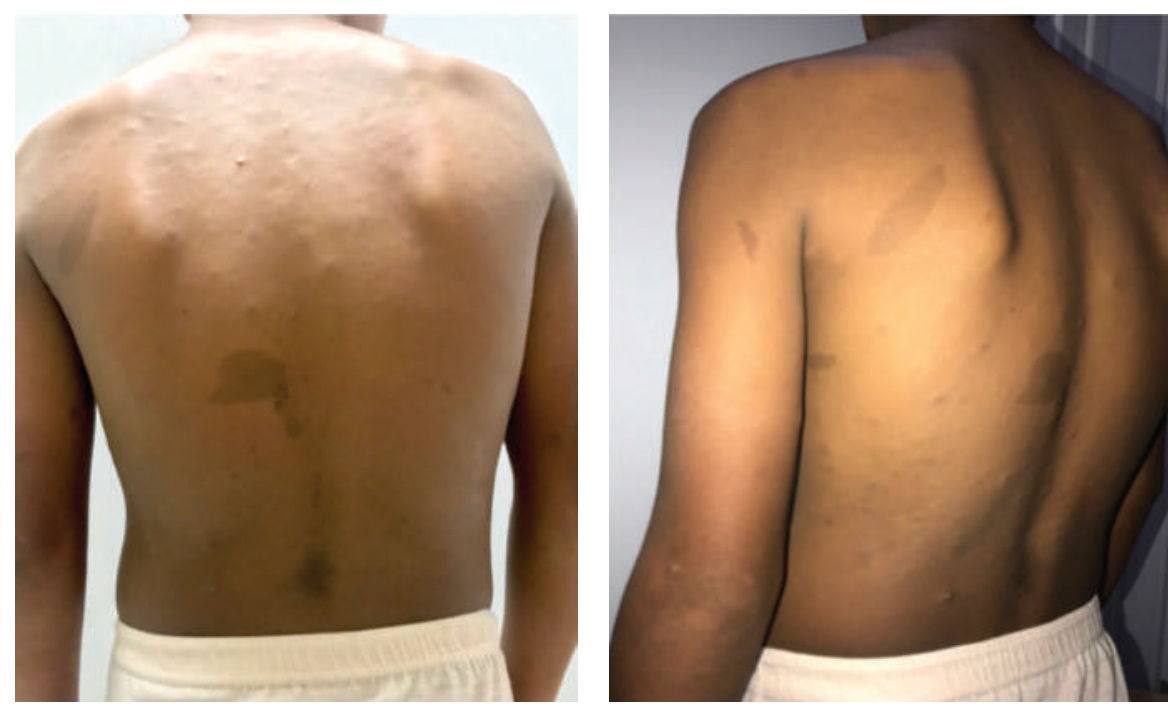

Figure 3 and 4. There were multiple brown macules and patches located on the arms, abdomen and back.

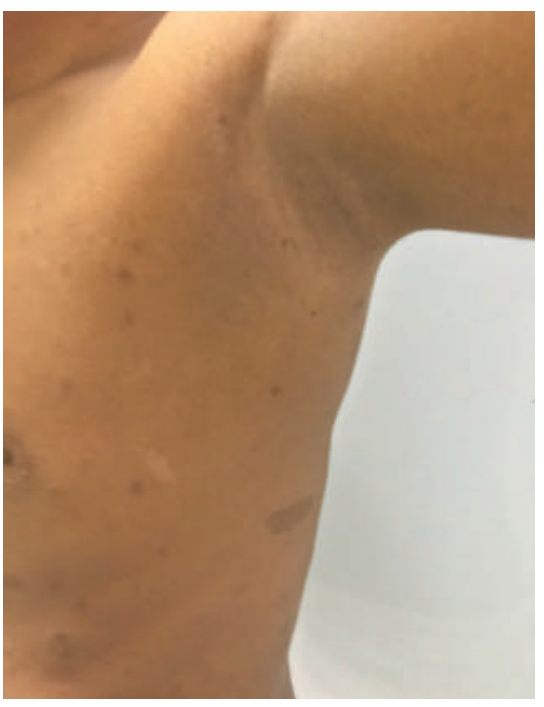

Figure 5. There was note of axillary freckling. 


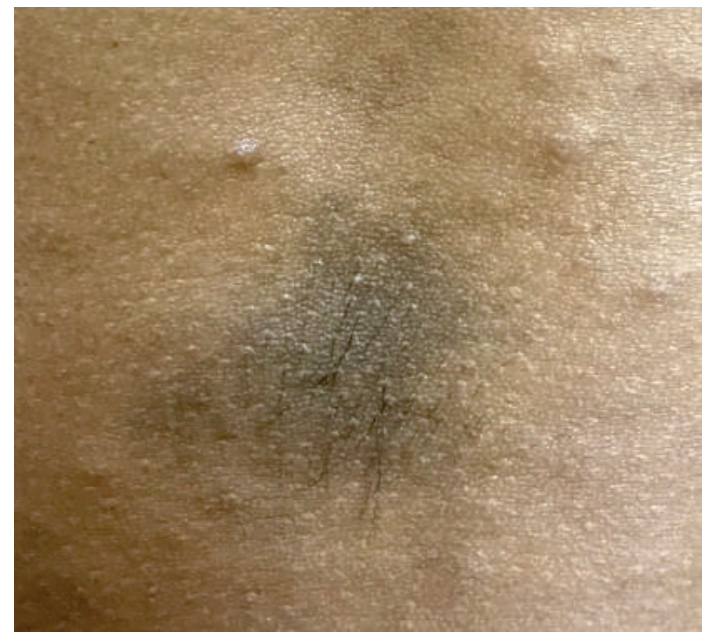

Figure 6. The black patch with hypertrichosis at the lumbosacral region.

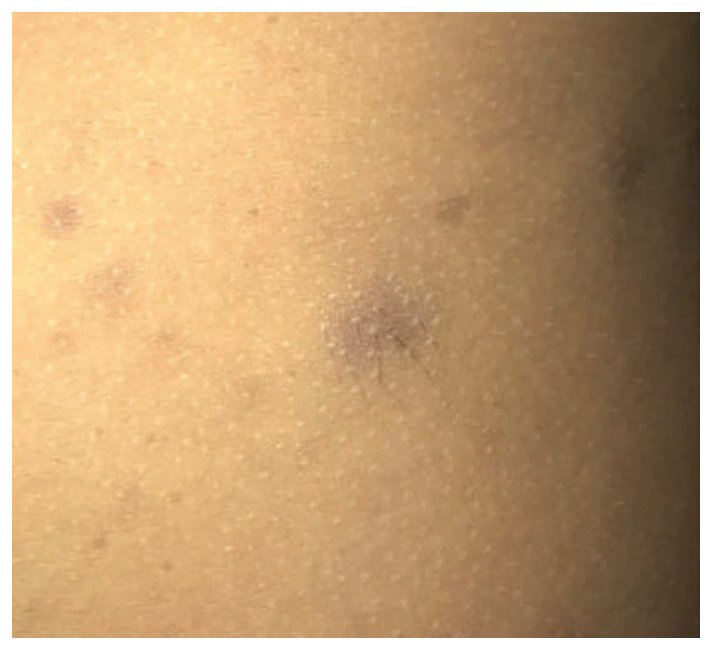

Figure 8. There were brown, slightly erythematous macules with hypertrichosis.

The patient was referred to the Pediatric service and they agreed with the diagnosis of NF 1 . He was assessed to have an intellectual disability based on the following: the patient was unable to recite the alphabet, do simple calculations, read simple words, and write dictated words. He was also assessed to have poor abstract thinking,and judgment. Ophthalmology noted multiple Lisch nodules on both eyes but did not identify any optic gliomas. No further imaging studies were recommended and no intervention was necessary for the current status. Radiographic examination showed thoracic dextroscoliosis and lumbar leftward asymmetry but no lytic changes or changes suggestive of underlying masses were noted.

The diagnostic criteria by the National institutes of Health Consensus Development Conference $1988^{2}$ require the fulfillment of two or more diagnostic criteria to

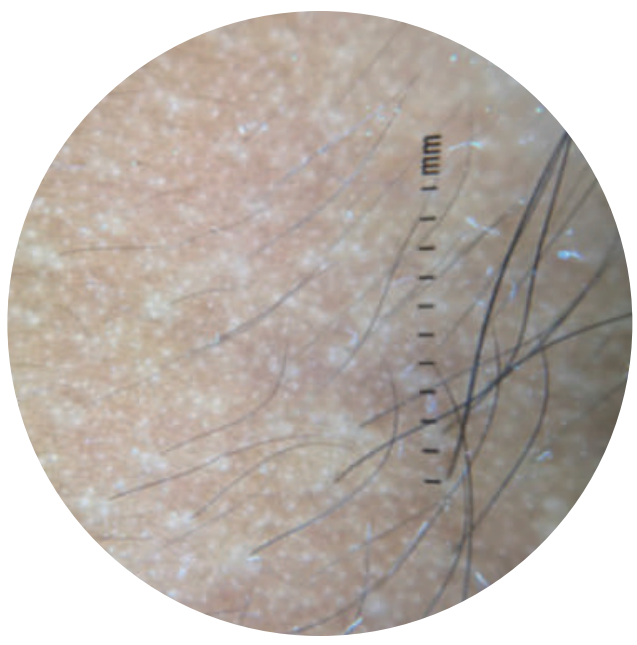

Figure 7. Dermoscopy of the lesion showed a brown reticular pattern with perifollicular hypopigmentation and growth of terminal hair.

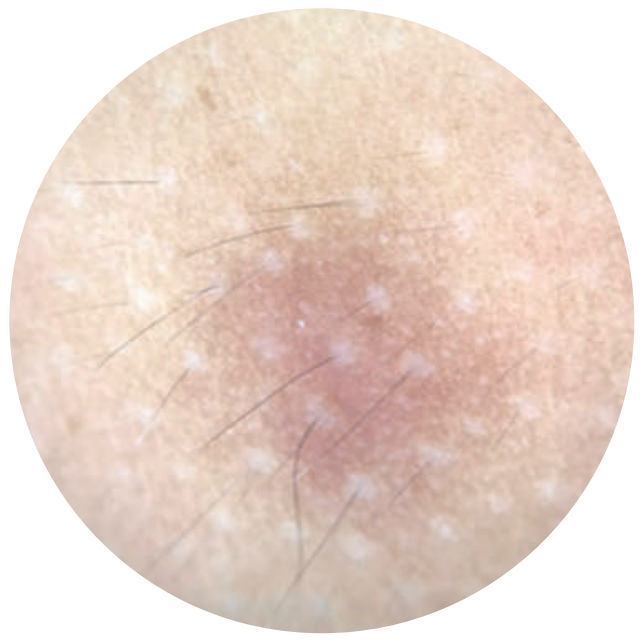

Figure 9. Dermoscopy of the lesion also revealed a brown reticular pattern with perifollicular hypopigmentation but with some areas of erythema and terminal hair.

establish the diagnosis of NF 1 . Since the patient had more than six café-au-lait macules five millimeters and greater in size, greater than two neurofibromas, axillary freckling, and Lisch nodules, a definitive diagnosis was made. Moreover, scoliosis, impaired vision, and learning disability are findings attributable to NF 1.

The patient and his guardian were educated about the nature and prognosis of the condition and the importance of regular follow-up to detect the development of complications and to address them accordingly.

\section{DISCUSSION}

Neurofibromas with hypertrichosis are not commonly seen. Reports of such cases showed lesions that occurred singly and localized to the head and neck region and 

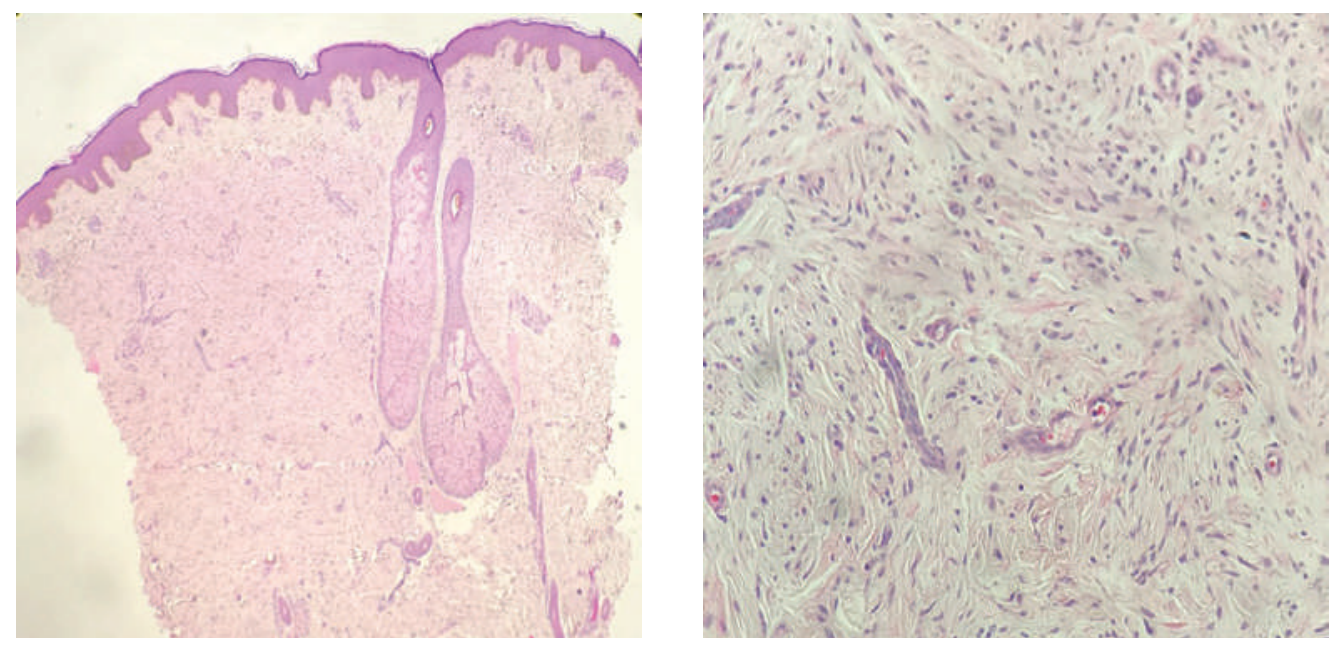

Figure 10. H\&E staining of the punch biopsy on a skin-colored nodule (scanning view and 20x magnification) that showed a well-circumscribed, unencapsulated proliferation of spindle-shaped cells with wavy nuclei in a pale mucinous stroma.
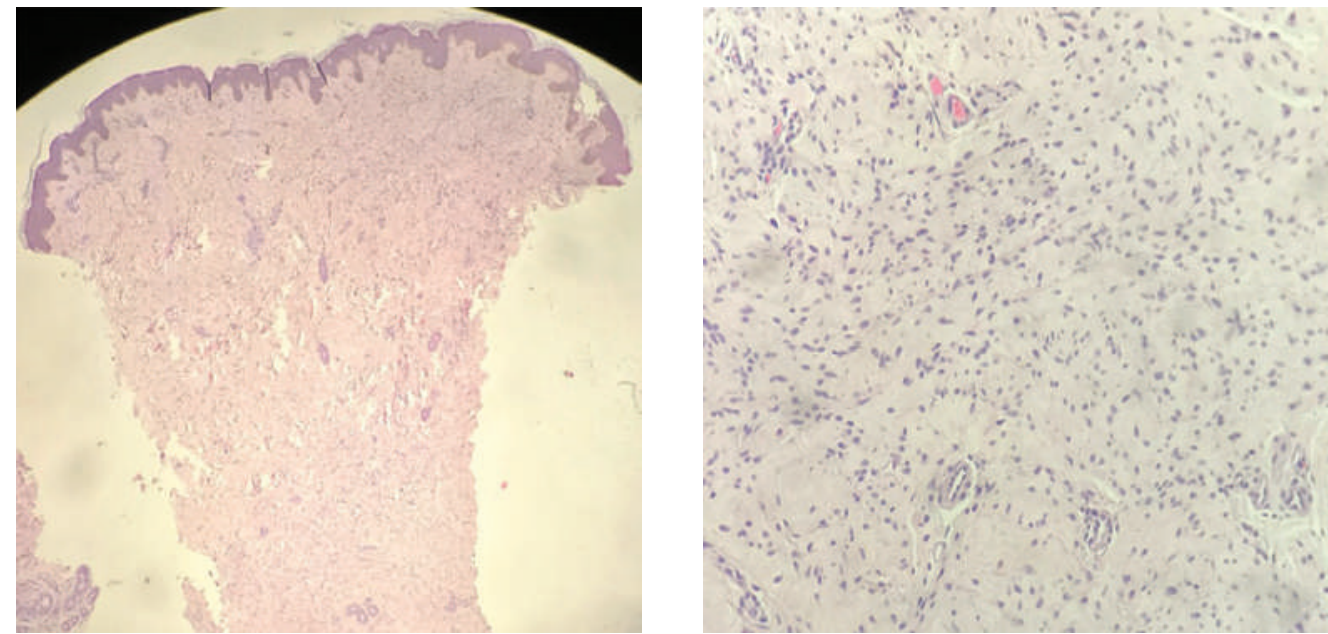

Figure 11. H\&E staining of the punch biopsy the black patch at the lumbar region (scanning view and 20x magnification) that also showed a well-circumscribed, unencapsulated proliferation of spindle-shaped cells with wavy nuclei in a pale mucinous stroma.
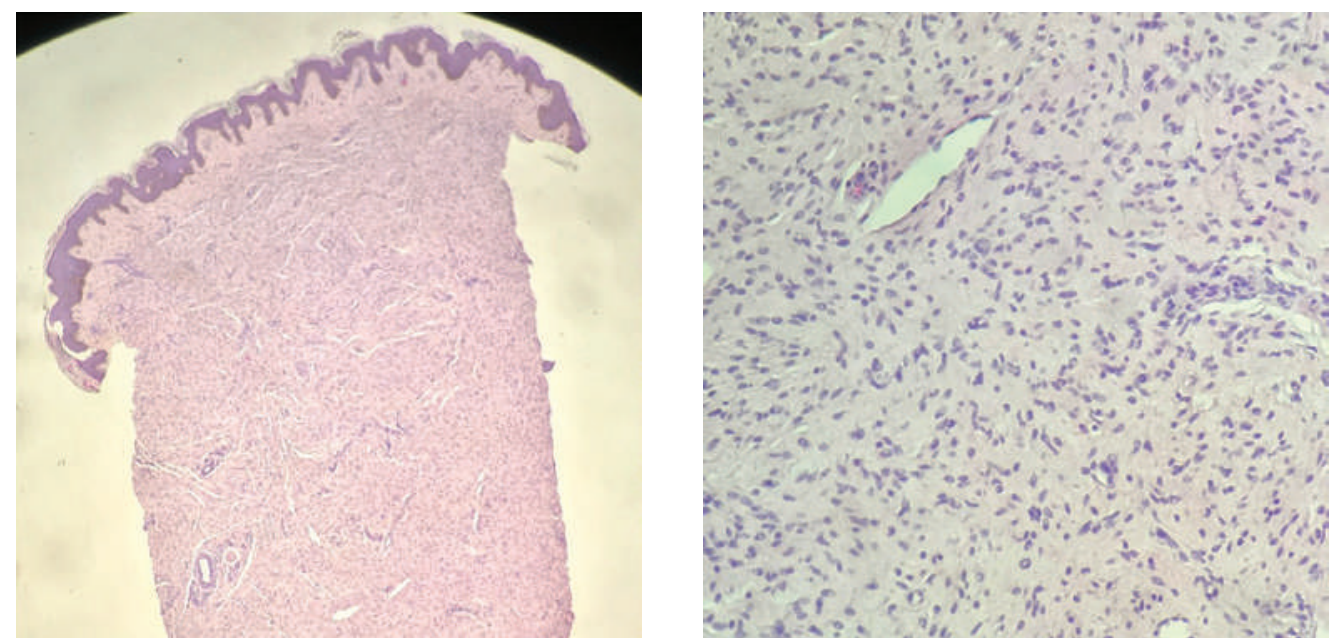

Figure 12. H\&E staining of the punch biopsy the brown macule with hair (scanning view and 20x magnification) still showed a well-circumscribed proliferation of spindle-shaped cells with wavy nuclei in a pale mucinous stroma. 
Table 1. The National Institutes of Health Consensus Development Conference 1988 diagnostic criteria for Neurofibromatosis 1 Six or more café-au-lait macules over $5 \mathrm{~mm}$ in greatest diameter in pre-pubertal individuals and over $15 \mathrm{~mm}$ in greatest diameter in postpubertal individuals.

Two or more neurofibromas of any type, or one plexiform neurofibroma

Freckling in the axillary or inguinal regions

Optic glioma

Two or more Lisch nodules (iris hamartomas)

A distinctive osseus lesions such as sphenoid dysphasia or thinning of long bone cortex, with or without pseudoarthrosis

A first degree relative with NF 1 by the above criteria

Two or more of the criteria have to be present in order to fulfill a diagnosis of Neurofibromatosis 1.

Criteria in boldface were present in the patient reported.

Table 2. Summary of studies on hypertrichosis and neurofibromas

\begin{tabular}{|c|c|c|c|}
\hline & & \multirow{2}{*}{$\begin{array}{l}\text { Presentation } \\
\text { Hyperpigmented macule with hypertrichosis overlying a swelling } \\
\text { from the right upper lid towards the forehead with proptosis }\end{array}$} & \multirow{2}{*}{$\begin{array}{l}\text { Other clinical findings of NF } 1 \\
\text { Axillary freckling } \\
\text { Cafe au lait macules } \\
\text { Lisch nodules } \\
\text { Learning difficulties }\end{array}$} \\
\hline \multirow{2}{*}{ Ettl and Koornneef (1995) } & & & \\
\hline & $\begin{array}{l}\text { Age/Sex } \\
14 / \mathrm{F} \\
12 / \mathrm{F}\end{array}$ & Hypertrichosis on the left half of face with worsening lid swelling & None \\
\hline Wagamon and Mirmirani (2007) & $7 / \mathrm{M}$ & $\begin{array}{l}\text { Area of excessive hair growth on the forehead that blended with } \\
\text { the anterior hairline with underlying subcutaneous fullness }\end{array}$ & $\begin{array}{l}\text { Axillary freckling } \\
\text { Café-au-lait macules } \\
\text { Cutaneous neurofibromas } \\
\text { NF 1-mother and grandfather }\end{array}$ \\
\hline $\mathrm{Na}$ et al (2009) & $17 / \mathrm{F}$ & Light brown plaque with terminal hairs on the post-auricular area & None \\
\hline Cho (2012) & $10 / \mathrm{F}$ & Brown plaque with terminal hairs on the posterior neck & None \\
\hline
\end{tabular}

occurred among the pediatric age group (Table 2). ${ }^{3-6}$ In reported studies, the area with hypertrichosis signified an underlying plexiform neurofibroma. Some cases presented in association with NF 1 while others were sporadic. The current study is the first report of multiple neurofibromas with hypertrichosis in the Philippines.

The mechanism for hypertrichosis in association with neurofibromas remains to be elucidated. A study exploring the pathogenesis of cutaneous neurofibromas showed that these lesions contain multipotent precursor cells which may be derived from the multipotent cells of the hair roots, that are intimately associated with microscopic neurofibroma. ${ }^{1}$ Given their spatial proximity, signals that stimulate the development of cutaneous neurofibromas may also stimulate follicular induction.

\section{CONCLUSION AND RECOMMENDATIONS}

The multiple plaques with hypertrichosis are atypical presentations of neurofibromas. Punch biopsy confirmed the etiology of the lesions that were previously managed as common dermatoses. The diagnosis of NF 1 also provided a parsimonious explanation for the constellation of findings of café-au-lait macules, neurofibromas, axillary freckling, impaired vision, learning disabilities, and scoliosis.

Therefore, in a patient presenting with localized hypertrichosis, an underlying neurofibroma may be considered. Moreover, investigation of other clinical findings associated with neurofibromatosis must be carried out.

\section{Statement of Authorship}

All authors approved the final version submitted.

\section{Author Disclosure}

All authors declared no conflicts of interest.

\section{Funding Source}

This paper was funded by the author. No external funding agency.

\section{REFERENCES}

1. Jouhilahti E, Peltoner S, Callens T, Jokinen E, Heape A, Messiaen L, and Peltonen, J. The Development of Cutaneous Neurofibromas. Am J Pathol. 2011;178(2):500-5. doi: 10.1016/j.ajpath.2010.10.041.

2. National Institutes of Health, Neurofibromatosis: consensus statement. [Online].1987 [cited 2018 Oct]. Available from https:// consensus.nih.gov/1987/1987Neurofibramatosis064html.htm .

3. Ettl A, Marinkovic M, and Koorneef L. Localized hypertichosis associated with periorbital neurofibroma. Ophthalmology, 103(6), 942-8.

4. Wagamon K, and Mirmirani P. Localized hypertrichosis in a pediatric patient - what is the mechanism for excess hair growth? Pediatr Dermatol. 2007;24(3):250-2.

5. Na CH, Song IG, Chung BS and Shin BS. Case of pigmented neurofibroma with hypertrichosis with no association to neurofibromatosis. J Dermatol. 2009;36(10):541-4. doi: 10.1111/ j.1346-8138.2009.00698.x.

6. Cho E, Kim HS, Lee JY, Kim HO, and Park YM. Localized hypertrichosis overlying neurofibroma. Int J Dermatol. 2013;52(12): 1623-4. doi: 10.1111/j.1365-4632.2011.05392.x. 